\title{
EL REFERENTE DE LA INTERNACIONALIZACIÓN Y SUS INHERENCIAS PARA LA EDUCACIÓN SUPERIOR PÚBLICA
}

\author{
Luis Muñoz Varela \\ Funcionario administrativo de la \\ Universidad de Costa Rica
}

Recibido 30-VIII-2004 • Aceptado 7-IX-2004

\begin{abstract}
Resumen: Se aborda en este artículo el tema de la internacionalización como dimensión de política y programática en el marco de la educación superior. Tal abordaje se lleva a cabo a partir de una amplia revisión bibliográfica realizada a propósito de resultados de investigación relacionados con el desarrollo, durante los últimos años, de esta dimensión de la política y de los procesos académicos, en instituciones y sistemas de educación superior de distintos países y regiones del mundo.

El artículo sustenta un anclaje como punto de partida en las implicaciones que la sociedad del conocimiento y la globalización establecen para la educación superior, por una parte; por la otra, identifica los ámbitos académicos y de la gestión institucional, en los que predominantemente la dimensión de internacionalización ha tendido a adquirir significado como parte de su incorporación en la política y en las disposiciones programáticas de las instituciones de educación superior.
\end{abstract}

Palabras clave: Internacionalización, educación superior, globalización, sociedad del conocimiento, intercambio académico, planes de estudio, redes académicas, gestión.

\section{Presentación}

Interesa aquí como propósito dar enunciación a un conjunto discreto de elementos que, bajo la óptica de una consideración crítica, permitan abonar a la discusión en el contexto del actual debate en el que las comunidades académicas y las instituciones de educación superior están inscritas, como resultado de las nuevas coordenadas de política y programáticas que para ellas derivan de las tendencias y procesos de la globalización y de la sociedad del conocimiento.

El tema de la internacionalización, como vector de política y como dispositivo programático, ha tenido en la educación superior una recepción y un anclaje difusos. En las instituciones de educación superior pública, también hace brotar una cierta reacción de aprehensividad, en cuanto se le enuncia como propuesta y como nuevo contenido de política para la gestión institucional de los procesos académicos. Casi podría afirmarse que, por ser este un tema asociado a las especificidades de la globalización que describen las trayectorias de cambio y de reordenamiento de 


\begin{abstract}
This article approaches the subject of internationalization as a dimension of programs and politics in higher education. For the purposes of this study, an extensive bibliography on the results of previous research studies was reviewed. The studies deal with this dimension of political and academic processes. The studies were carried out in the recent years in institutions and systems of higher education in different regions and countries of the world. As point of departure, the article discusses the implications that the society of knowledge and globalization have on higher education. It also identifies the academic and the institutional scopes in which the dimension of internationalization has been more meaningful as a result of its incorporation in politics and in the programmatic endeavors of institutions of higher learning.
\end{abstract}

Key words: Internationalization, higher education, globalization, society of knowledge, academic exchange, study plans, academic networks, management. los sistemas económicos en clave de libre mercado o de aplicación sine qua non del principio de la competitividad, la internacionalización configura una especie de identidad sospechosa cuyos emblemas no ofrecen ni mayor confianza ni tampoco una legibilidad transparente.

Sin embargo, para las instituciones de educación superior, la internacionalización tampoco deja de ser un tema que, en todo caso, genera un interés cada vez más creciente en diferentes países y regiones del mundo. Como tal, en su vinculación académica, la internacionalización se promueve como un escenario a partir del cual la educación superior dispone de un espacio emergente, que puede ser asumido apropiadamente como un vector de política y para el desarrollo de acciones académicas institucionales, en perspectiva de abrir y constituir nuevos espacios y escenarios para el relacionamiento y la cooperación interinstitucionales.

Este artículo es el comprimido de una reflexión desarrollada en el marco de mi proyecto de tesis de maestría: La educación superior pública en Centroamérica: fricciones y convergencias entre la integración regional y la internacionalización. El proyecto fue realizado en el Departamento de Investigaciones Educativas del Centro de Investigación y Estudios Avanzados (CINVESTAV) del Instituto Politécnico Nacional (México D. F.), bajo la dirección de la Dra. Sylvie Didou Aupetit y con la participación como sinodales del Dr. Eduardo Remedi Allione (DIE/CINVESTAV) y Dr. Roberto Rodríguez Gómez (Centro de Estudios sobre la Universidad, Universidad Nacional Autónoma de México, UNAM).

\section{Introducción}

La característica reciente del mundo de ser comunicado y vivido electrónicamente, en dimensión virtual, presenta muy diversas implicaciones culturales, societales e institucionales. Una de ellas, especial 
y genéricamente relevante, está dada por la emergencia de una definición de "lo real", que ahora pone en literal condición crítica a todos los anteriores sentidos con que en el pasado fueron desplegados y asumidos los entramados de configuración vital de lo cotidiano, y que daban su sustento a la estabilidad de las "totalidades socioculturales y políticas.” (Bauman, 2001, p. 24).

Diferentes expresiones y con diferente acepción, durante los últimos años han sido acuñadas para intentar categorizar los fundamentos estructurantes que otorgan su cualidad específica al nuevo momento histórico que, especialmente a partir de la década de los '90, adquirió la denominación de 'globalización'. Expresión esta que tiene su antecedente ya en los escritos de McLuhan de los años '70, cuando este autor conceptualizaba las implicaciones de los procesos generados en las sociedades altamente industrializadas como de configuración de una condición de interrelaciones planetarias que cabía denominar en términos de 'aldea global'. (Valaskakis, 1999, p. 4).

Para un segmento mayoritario de los actores de la educación superior, el concepto y referente de la internacionalización, tal vez no haya logrado aún adquirir en absoluto mayor significación. Se trata de un concepto que todavía presenta una representación difusa en los imaginarios de las colectividades académicas de muchas instituciones de educación superior. No obstante, existe también al menos un pequeño segmento de estos actores para quienes la internacionalización, no sólo es un tema conocido, sino que representa uno de los vectores emergentes de política y de gestión más importantes, a partir de las cuales ahora se articula el proyecto académico y de formación en estas instituciones.

La identificación de la internacionalización, en todo caso, hace delimitar uno de los espacios de la gestión desde los que se originan dispositivos para una reasunción innovadora de los procesos de desarrollo académico y de las estrategias del cambio institucional. La internacionalización admite ser relevada y se propone así como un referente que aporta un interés programático significativo, en el contexto de los procesos de cambio que las instituciones de educación superior han venido impulsando durante los últimos años. Alcanza a establecer una relación significativa con los procesos de reforma de los planes y programas de estudio, la redefinición pedagógico/didáctica del proyecto de formación, la gestión de la investigación y el posgrado, el fortalecimiento de la gestión institucional, etc. Todas estas dimensiones, en su conjunto y articuladas con la política, programas y proyectos de internacionalización, conducen a procesos que se orientan al mejoramiento de la calidad (Gacel, 2000a, p. 25) y a la búsqueda de la excelencia académica.

En su acepción académica de mayor implicación, la internacionalización se ofrece como la posibilidad de una nueva modalidad de relacionamiento interinstitucional y de articulación programática del proyecto de desarrollo académico e institucional. Su establecimiento como política y como incidencia programática propone muy diversos despliegues y avances, según la región o países del mundo. Así, por ejemplo, la Universidad Uppsala de Suecia, la primera universidad en el mundo en establecer en 1630 un programa de ciencias políticas (Barnett, 2001, p. 57), cuenta ya con un modelo de internacionalización desde hace más de tres lustros (Fransson, 2000, p. 20).

En su caso, no obstante, en América Latina la mayoría de las instituciones de educación superior aún registran una situación en la que, en sus políticas y programas, las actividades que se proponen y llevan a cabo con frecuencia no involucran "un plan de internacionalización con políticas y estrategias claramente expresadas con objetivos y metas a largo plazo." (Boyle, 2000, p. 12). La internacionalización constituye, en América Latina, todavía en muchos casos un tema apenas ajeno. Y ello es así, cuanto más que los referentes 
prevalecientes de la gestión académica e institucional, cristalizados en los esquemas de lo prosaico y lo tradicional, enrarecen la posibilidad de su incorporación en las políticas y en los cursos de acción en los que se ubican y adquieren su horizonte de sentido los actuales procesos de desarrollo académico e institucional.

Este panorama y la situación que da cuenta de él no son casuales. En su generalidad, revela la diferencia de desarrollo que la internacionalización presenta privilegiadamente en la educación superior de los países del Norte -e incluso en otras regiones del mundo, Australia o Nueva Zelanda-, en comparación con las condiciones que en este sentido caracterizan el comportamiento de la educación superior pública en América Latina. Una razón para esto, seguramente habría que atribuirla a la existencia de una mayor disposición por parte de los gobiernos de aquellos países para brindar apoyo a sus instituciones educativas.

La búsqueda permanente y sistemática de la excelencia académica e institucional es percibida en tales países como un elemento de implicación vital, y que toma mayor preponderancia en el marco de la globalización y de la sociedad del conocimiento. Los gobiernos, por consiguiente, buscan proporcionar apoyo y favorecer las condiciones que coadyuven al fortalecimiento de los procesos de desarrollo académico y de la proyección internacional de sus instituciones educativas. (OECD, 2004, p. 9). Tal disposición, en su escala macrorregional, ha sido generadora de megaproyectos con una amplísima proyección de cobertura. Es este el caso, por ejemplo, del así denominado América Latina Formación Académica (ALFA), instituido en 1994 para la constitución de redes académicas internacionales entre universidades de Europa y América Latina; un programa que alcanza una cobertura en una muy diversa gama temática y en múltiples ámbitos del desarrollo académico: gestión, investigación, posgrado, planificación estratégica, relación universi$\mathrm{dad} /$ sector productivo, etc.
La iniciativa de ALFA es claramente ejemplificadora de las estrategias de proyección que, en el marco de la globalización y de la sociedad del conocimiento, ponen en articulación las políticas públicas estatales en relación con la educación superior. Como tal, ALFA constituye una cualificación de escala de las políticas y programas de la Unión Europea dirigidas al fortalecimiento de la educación superior, las cuales alcanzan de este modo una proyección macrorregional.

Los antecedentes de ALFA están dados por el Programa Sócrates, experiencia de integración regional europea en el campo educativo iniciada en 1976 (Hernández, 2002, p. 4), y cuyo establecimiento en su formulación actual fue decidida en 1995 por el Parlamento Europeo y el Consejo de la Unión Europea, bajo la orientación de "contribuir al desarrollo de una enseñanza y una formación de calidad y de un espacio europeo abierto de cooperación en materia de enseñanza." (IPE, 2005, p. 1). Entre otros, SOCRATES integra un capítulo para la educación superior, el Programa ERASMUS, el cual presenta como sus acciones principales la promoción de la dimensión europea en las universidades y el fomento de la movilidad de los estudiantes universitarios y la financiación de becas. (IPE, 2005, p. 2).

Para todos es un hecho que la excelencia académica, como concepto matriz fundante y en tanto horizonte del proyecto de formación en la educación superior, adquiere un lugar privilegiado y cada vez más connotado a partir de los procesos que la globalización y la sociedad del conocimiento establecen. En una de sus expresiones particulares, las preocupaciones y complejidades que de ello derivan las pone de relieve, por ejemplo, el estudio comparativo de la situación que involucra a la educación superior de México, en relación con la de los otros dos países signatarios del Tratado de Libre Comercio de América del Norte (TLCAN). En ese escenario, toma su visibilización una situación en la que, paradigmáticamente, devienen críticas las 
condiciones de asimetría que caracterizan el relacionamiento entre las instituciones educativas de uno y otros países. En este caso, tales asimetrías tienden a esbozar una situación considerada como desventajosa en perjuicio de las instituciones de educación superior mexicanas. (Mungaray y Ocegueda, 1998, pp. 200/206).

Con todo y, precisamente por la misma razón por la que se afronta tal tipo de situaciones de asimetría, la internacionalización deviene como una opción de política y como una alternativa de acción que, de un modo u otro, adquiere cada vez mayores y más complejos grados de interés en el seno de las comunidades académicas y en los contextos de la gestión, independientemente del país y de la región geográfica en la que estas instituciones se encuentren ubicadas.

\section{Internacionalización de la educación superior}

\section{II.1. Especificidades genéricas}

Las consideraciones que derivan de los procesos en los que las instituciones de educación superior están inscritas como resultado de la globalización y de la sociedad del conocimiento hacen emerger el señalamiento de que: "A la hora de la apertura comercial, a la hora de la transformación del Estado, a la hora de la revolución científica y tecnológica, es imperativo establecer nuevos mecanismos de interacción" (Van Der Donckt, 1997, p. 58) entre las comunidades académicas y los diferentes actores involucrados e interesados en el quehacer de la educación superior. A propósito de ello, cabría agregar, a título de observación inquietante, una cierta lapidaria afirmación enunciada por A. Robinet hace ya varios años: "En todo sistema aislado la entropía crece." (Robinet, 1982, p. 185).

Estas inquietudes y preocupaciones prefiguran, en la dimensión de las relaciones interinstitucionales, el vértice de entrada quizá privilegiadamente significativo, que interpela y convoca a la asunción de los procesos, políticas y estrategias de internacionalización de la educación superior.

La era planetaria necesita situar todo en el contexto y en la complejidad planetaria. El conocimiento del mundo, en tanto que mundo, se vuelve una necesidad intelectual y vital al mismo tiempo. Es el problema universal para todo ciudadano del nuevo milenio: ¿cómo lograr el acceso a la Información sobre el mundo y cómo lograr la posibilidad de articularla y organizarla? ¿Cómo percibir y concebir el Contexto, lo Global (la relación todo/partes), lo Multidimensional, lo Complejo? (Morin, 2001a, p. 35).

La educación superior, sus políticas, estrategias de gestión y su proyecto académico, se inscriben de este modo en la situación imperativa de llevar a cabo reformas académicas, que describen y trazan repercusiones estructurales para el proyecto de formación. En todo caso y, de un modo $\mathrm{u}$ otro, estas instituciones han debido dar curso en el transcurrir de los últimos años a acciones diversas en tal sentido, aún cuando estas mismas acciones puedan o no aparecer explícitamente definidas o categorizadas como tales.

En América Latina, las reformas impulsadas han estado asociadas predominantemente a los procesos de la globalización económica. (García Guadilla, 2003, p. 17).

Pero por otra parte, en una de sus dimensiones programáticas de relieve, las propuestas de reforma impulsadas han tenido como su referente fundamental la consideración de que, con la globalización y la sociedad del conocimiento, la transformación de los perfiles de la formación deberá ahora abocarse, no sólo a aportar una formación sustentada en las habilidades y los conocimientos propios de cada especialidad; antes bien y, de igual manera, deberán hacerlo en función de una formación diversa e integral.

Según lo anterior, los nuevos profesionales han de contar con una formación que los capacite para situarse apropiadamente en los contextos laborales y de la producción con disposición creativa y 
crítica; perfiles profesionales definidos por el dominio de las habilidades que ahora hacen necesarios los procesos cada vez más intensos de la innovación científica y tecnológica. (Feldman, 2002, pp. 61/62). $\mathrm{Al}$ propio tiempo y, en una dimensión cada vez más relevante, que los nuevos profesionales sean también personas conscientes de la interdependencia que caracteriza a las sociedades del mundo contemporáneo; es decir, titulados con una formación sustentada en una sólida sensibilidad ética y con sentido de la pertinencia que cada vez más adquiere la tolerancia y el respeto a la diversidad cultural. (Conferencia Mundial sobre la Educación Superior/UNESCO, 1998 , p. 10 y 18).

El mundo en el que nos toca vivir es ya un mundo global en el que todo está relacionado, tanto nacional como internacionalmente; un mundo donde las dimensiones financieras, culturales, políticas, ambientales, científicas, etc., son interdependientes, y donde ninguno de tales aspectos puede ser adecuadamente comprendido al margen de los demás. Cualquier toma de decisiones en alguna de esas parcelas debe conllevar una reflexión acerca de las repercusiones y los efectos colaterales que cada una tendrá en los restantes ámbitos. (Torres, 2000, p. 31).

En una perspectiva de orientación estratégica y de vinculación con la sociedad, la pauta de la transformación en la educación superior configura una directriz que desde las matrices complejas de la globalización y de la sociedad del conocimiento, convoca a estas instituciones a incorporar en sus procesos de desarrollo, lineamientos acordes con requerimientos de mayor consideración hacia la naturaleza inter y multicultural que ha de ser propia de todo proyecto educativo.

El problema de la integración de la vida cultural se convierte en el problema de hacer posible que las personas que habitan en mundos distintos tengan un impacto genuino y recíproco unos sobre otros. Si es cierto que, en la medida en que exista una conciencia general, ésta ha de consistir en la interacción de una caótica multitud de visiones no del todo conmensurables, entonces la vitalidad de esa conciencia dependerá de la creación de las condiciones bajo las que pueda darse esa interacción. (Geertz, 1984, p. 190).
Recientemente, la Conferencia Mundial sobre la Educación Superior de la UNESCO definió la educación superior como un escenario de naturaleza pluridimensional, caracterizado por "el intercambio de conocimientos, la creación de sistemas interactivos, la movilidad de profesores y estudiantes y los proyectos de investigación internacionales". (Conferencia Mundial sobre la Educación Superior/UNESCO, 1998, p. 11). Tal realidad pluridimensional y compleja ha de tener a su vez su anclaje privilegiado en los referentes de historicidad que en cada caso devienen prefigurados por "los valores culturales y las situaciones nacionales" específicas de cada país y de cada contexto institucional.

Desde América Latina, en la óptica de la CEPAL y según los enunciados que se ofrecen en el planteamiento de E. Ottone:

Se trata pues de generar una educación que prepare para la vida en un proceso productivo cambiante, menos jerárquico, y más basado en una organización reticular, con carreras que no serán lineales y cuyas fronteras no serán las de un país sino las del mundo, donde se requerirá más iniciativa que docilidad, más creatividad que orden. (Ottone, 1998).

En la perspectiva de una 'educación para el futuro' y conforme al enunciado del principio de la "unitas multiplex", É. Morin, colaborador de la UNESCO, propone una visión de la educación y de su organización y orientación programática, en función de "velar porque la idea de unidad de la especie humana no borre la de su diversidad, y que la de su diversidad no borre la de la unidad." (Morin, 2001b, p. 53).

Este planeta necesita un pensamiento policéntrico capaz de apuntar a un universalismo no abstracto sino consciente de la unidad/diversidad de la humana condición; un pensamiento policéntrico alimentado de las culturas del mundo. Educar para este pensamiento; esa es la finalidad de la educación del futuro que debe trabajar en la era planetaria para la identidad y la conciencia terrenal. (Morin, 2001b, p. 62).

\section{En otra formulación:}

La diversidad de las culturas, la diversidad de los individuos entre sí, la diversidad interior de los 
individuos no pueden comprenderse ni a partir de un principio simple de unidad, ni a partir de una plasticidad muelle modelada por las culturas a merced de las circunstancias. (Morin, 2003, p. 73).

Así, tomando en consideración los anteriores enfoques y enunciados, la internacionalización de la educación superior puede ser definida como un eje transversal de política institucional, desde el que se generan proyectos y estrategias cuya articulación programática y de gestión se dirige a contribuir con una mejor visualización de lo que, en el presente, constituye el proyecto de desarrollo académico en la educación superior.

La internacionalización, en su acepción académica y en su específica articulación como tal, supone una dimensión del quehacer académico e institucional orientado a contribuir transversalmente con el fortalecimiento, ampliación y diversificación de la visión de mundo y de las disposiciones comprehensivas en general de los estudiantes.

Esta perspectiva involucra no sólo el desarrollo de un quehacer académico asumido como el escenario de la enseñanza de las habilidades y destrezas relativas a los requerimientos del mercado de trabajo y del aparato económico/productivo. Consiste, en su lugar y de manera privilegiada, también en un soporte que contribuye a la constitución de un espacio académico e institucional de formación, caracterizado por un escenario curricular sustentado en la promoción de la creatividad, la imaginación, la sensibilidad ética, el compromiso social, tanto como en la consideración y respeto hacia las diferencias étnicas, de género, intergeneracionales, etc.

En suma, una educación fluida e interactiva que cree una mente escéptica, curiosa y creativa. (...) Todo el esfuerzo de la transformación educativa para responder a un futuro que sea moderno, democrático y sustentable será el de no hacer de la competitividad sinónimo de barbarie y exclusión ni de la solidaridad sinónimo de pasividad e ineficiencia. (Ottone, 1998).

La internacionalización, incorporada como un componente para el fortalecimiento del proyecto académico institucional, se encuentra por lo tanto asociada a una proyección pedagógica que busca desarrollar la formación en la perspectiva axiológica de la convivencia y por el reconocimiento mutuo de la diversidad entre los pueblos y las culturas. En la óptica de la UNESCO, esto constituye precisamente uno de los cuatro pilares en los que en la actualidad se resignifican las bases de la educación y, en particular, de la educación superior.

Se trata de aprender el aprender a vivir juntos conociendo mejor a los demás, su historia, sus tradiciones y su espiritualidad y, a partir de ahí, crear un espíritu nuevo que impulse la realización de proyectos comunes o la solución inteligente y pacífica de los inevitables conflictos, gracias justamente a esta comprensión de que las relaciones de interdependencia son cada vez mayores y a un análisis compartido de los riesgos y retos del futuro. (Delors, 1996, p. 18).

A propósito de sus finalidades, es importante recalcar asimismo, que la internacionalización remite a un proyecto académico dirigido a la transformación y al despliegue propositivos de las funciones sustantivas que dan su naturaleza característica a las instituciones de educación superior. En este sentido, se considera asimismo y valora como una finalidad de la internacionalización, el garantizar la articulación solidaria del relacionamiento interinstitucional, con el fin de enriquecer y ampliar los mecanismos y las estrategias de la cooperación interuniversitaria, en una perspectiva de apoyo y de beneficio recíprocos.

\section{II.2. La internacionalización en las instituciones de educación superior}

El establecimiento de una política y de un programa de internacionalización en las instituciones de educación superior, involucra la asunción de la tarea por llevar a cabo procesos institucionales de incidencia amplia y diversa. En el análisis propuesto por S. Didou: 
(...) la promoción de una dimensión internacional en la educación supone una conjunción amplia de esfuerzos, de arriba hacia abajo y de abajo hacia arriba y una armonización básica entre los objetivos perseguidos por las políticas gubernamentales y por las institucionales. Asimismo, depende de una definición de las estrategias convenientes a largo, mediano y corto plazo, de una inversión financiera constante y etiquetada así como de la construcción de acuerdos mínimos entre los diferentes actores intra y extra universitarios involucrados. (Didou, 2000, p. 142).

La internacionalización reviste en la educación superior el carácter de una dimensión programática concreta y específica. Esto tiene lugar en el marco de una política institucional que busca organizar acciones acordes dirigidas a remover $\mathrm{y}$ cualificar tanto las formas tradicionales de realizar y atender la cooperación internacional e interinstitucional, cuanto a generar contribuciones en los escenarios de la reforma académica, el proyecto de formación a nivel de grado y posgrado, la evaluación y la acreditación y, en sus distintas especificidades, las directrices y mecanismos de la gestión académica e institucional, la investigación y la constitución de redes académicas internacionales.

Tal política de internacionalización, según lo señalado antecedentemente, deriva a su vez de al menos tres vectores.

El primero de estos es la sociedad del conocimiento. Los escenarios que la sociedad del conocimiento hace desembocar desprenden procesos que afectan y trastocan, por una parte, las formas tradicionales de realizar la investigación y de producir conocimiento científico/tecnológico en la educación superior; por la otra, la sociedad del conocimiento también origina nuevos enfoques pedagógico/didácticos, así como nuevas coordenadas curriculares para la formación de los estudiantes.

Un segundo vector está dado por los requerimientos del desarrollo nacional. En la actualidad, las condiciones del desarrollo nacional configuran la necesidad de una formación sustentada en perfiles profesionales cualitativamente diferentes, cifrados estos no sólo en los conocimientos y habilidades propios de las diferentes especialidades, sino también abocados a que los nuevos profesionales sean personas capaces de situarse apropiadamente en los actuales contextos laborales y de la producción de una manera creativa, crítica, con habilidades y disposición para la innovación.

Un tercer vector, lo constituyen las tendencias a la transnacionalización educativa que durante los últimos años se han impulsado, especialmente en el marco del Acuerdo General sobre Comercio de Servicios (AGCS) de la OMC. (Knight, 2003, p. 37). Tal como se sabe, en el escenario de la globalización, estas disposiciones y tendencias tienen su lugar en función de la apertura de opciones para el establecimiento y proliferación locales de ofertas de servicios educativos provenientes de muy diversas regiones y países del mundo.

Este último escenario, en su dimensión exorreferencial, está asociado a diversos elementos de naturaleza estructural a partir de los cuales hoy adquieren su articulación las realidades de la educación superior: procesos de integración regional, homologación de competencias profesionales, estándares de acreditación internacional para el reconocimiento de títulos y diplomas, evaluación y acreditación, oferta de servicios educativos en línea, etc.

No obstante, a diferencia de la disposición comercial que define a estas tendencias, el concepto de internacionalización no remite en este caso al imaginario y propuesta de una oferta académica transnacionalizada o con propósitos de ser colocada competitivamente en el mercado transnacional de los servicios educativos. Tal enfoque, tendencias y propuestas, requerirían ser denominadas antes bien y de manera apropiada como transnacionalización de la educación superior. Por su específica naturaleza, tal referente representa o comporta configuraciones de sentido y prácticas de proyección y de relacionamiento interinstitucional, distintas de lo que puede ser genuinamente entendido por internacionalización en el marco de la educación superior pública. 
En particular, teniendo en cuenta la naturaleza de los perfiles académicos e institucionales que caracterizan la educación superior pública (Consejo Universitario, 2004, p. 1) y, especialmente en el contexto de sociedades como las centroamericanas, la internacionalización no puede ser entendida sino como expresión de un ethos configurado a partir de los principios de la cooperación y la solidaridad, con sustento privilegiado en relaciones interinstitucionales de reciprocidad. Y así es precisamente como se entiende en el marco del ideario integracionista que da su horizonte al Segundo Plan de Integración Regional de la Educación Superior Centroamericana (PIRESC II), del Consejo Superior Universitario Centroamericano (CSUCA).

Promover una auténtica comunidad universitaria regional, que sea ejemplo de unidad y de solidaridad y que procure como meta la unión de América Central. La Confederación en una perspectiva regional, deberá apoyar los esfuerzos científicos y culturales conducentes a la integración latinoamericana; auspiciará además las relaciones con las universidades de todos los países del mundo y cimentará la convivencia y la paz universal a través de un diálogo permanente. (Consejo Superior Universitario Centroamericano, 2003).

Es este el sentido propositivo y aportante que puede retribuir el desarrollo de una política y de acciones de internacionalización en el marco de la educación superior.

La colaboración internacional es una dimensión constitutiva de la historia y de la identidad de las universidades, tal como se las concibe desde hace siglos. Más aun, los universitarios siempre han considerado a la cooperación con colegas de otras latitudes como fermento de innovación y portadora de mejores resultados. (Universidad de Lima, 2002).

Valga recalcar la necesidad de diferenciar lo que significa la internacionalización de la educación superior como una dimensión que propicia y establece condiciones para el desarrollo y fortalecimiento de relaciones cooperativas a nivel interinstitucional -y extrarregional o macrorregional- y, en otro sentido, las heurísticas de desarrollo que establece y los objetivos que promueve la transnacionalización de la educación superior. Uno y otro concepto deben ser significados de manera precisa en su específica diferencialidad.

\section{II.3. Internacionalización y breves puntualizaciones sobre el AGCS}

La Organización Mundial del Comercio (OMC) ha promovido durante los últimos años un enfoque y ha impulsado acuerdos encaminados a que la educación superior pase a formar parte como uno más de los bienes tipificados bajo la rúbrica de comercio de servicios, y que se integre paulatinamente al flujo de estos. La propuesta del organismo multilateral propugna por una apertura creciente de la comercialización de los servicios educativos (Chan, 2003, p. 32), especialmente en los renglones que corresponden a estudios en el extranjero, enseñanza impartida por docentes venidos del exterior, enseñanza a distancia de un país a otro, implantación de institutos extranjeros. (Internacional de la Educación, 2000).

No obstante, una política académica de internacionalización, esto es, definida desde el espacio institucional de la educación superior pública, difiere por naturaleza de lo que representan los movimientos promovidos, en este caso, por la OMC. Naturalmente y, por definición, en la educación superior pública no es la dimensión del comercio de servicios educativos la que en su contexto interesa relevar. La naturaleza de la educación superior pública es la de la educación como bien público. (Panizzi, 2003, p. 96). Así, en el caso de la internacionalización, de lo que se trata es de la articulación de una política y de la construcción de un espacio de acciones y de interactuación institucionales, en el que el postulado fundante ha de ser, en la perspectiva del respeto y la reciprocidad interinstitucionales, el de la consideración de las particularidades, de las diferencias culturales y de la historicidad propias que, en 
cada caso, identifican las diferentes instituciones. En otras palabras, una perspectiva programática y axiológica que privilegia como condición fundamental la valoración de que la cooperación interuniversitaria y la cooperación internacional en materia de educación superior, operan de manera más apropiada con base en los principios de la solidaridad y de la reciprocidad.

Al diferir los enfoques y las lógicas que delimitan y recortan los conceptos, lo que para las iniciativas de transnacionalización de la OMC constituyen "servicios", en la perspectiva académica de la internacionalización representa un conjunto organizado de actividades académicas susceptibles de ser desarrolladas en el marco de la cooperación interuniversitaria y de la cooperación internacional.

Con el mismo vigor y vehemencia se podría cuestionar si la internacionalización que se hace cargo plenamente de la diversidad y las diferencias entre culturas nacionales en verdad forma parte de, y ni hablar de que sea compatible con, la globalización y el cauteloso hegemonismo que prácticamente no se ocupan de otra cosa que no sea su propia extensión. (Neave, 2001, p. 12).

El enfoque que promueve la trasnacionalización de la educación superior, no se corresponde así en ninguno de sus extremos con lo que legítimamente puede ser entendido por internacionalización en el marco de la educación superior.

El término ‘internacionalización' se refiere al proceso de integrar la dimensión internacional a las funciones de docencia, investigación y servicio que desempeñan las instituciones de educación superior. Su uso ha estado más estrechamente relacionado con el valor académico de las actividades internacionales que con la motivación económica. De hecho, el término internacionalización sin ánimo de lucro' se ha acuñado recientemente para diferenciar la educación internacional del comercio de servicios educativos. (Knight, 2002, p. 3).

J. Knight es recurrente en destacar esta distinción, señalando algunas de las consecuencias de no diferenciar claramente entre las implicaciones de fondo inherentes a cada uno de ambos enfoques:
Es necesario prestar atención al impacto que tiene la liberalización del comercio en las actividades de internacionalización sin ánimo de lucro. ¿Llegará el comercio a opacar y dominar las relaciones académicas internacionales entre países e instituciones, o las fortalecerá? Muchas estrategias de internacionalización podrían quedar expuestas si se adopta un enfoque puramente comercial. (Knight, 2003, p. 60).

Y por su parte, García Guadilla llama la atención sobre lo que a su juicio constituyen dos dinámicas críticas que en este sentido afectan a la educación superior. Primero, la dinámica de los GATS y de los "mercados lucrativos" de la educación. Segundo, la dinámica de la crítica y del rechazo que se genera al interior de la educación superior contra la primera dinámica. Dos dinámicas polarizadas, ante las cuales las instituciones de educación superior se ven en la necesidad de desarrollar acciones conducentes a la identificación y asunción de alternativas.

Frente a estas dos dinámicas, la del mercado con su implacable agenda, y la de la crítica necesaria, es urgente, a nivel internacional, regional y nacional, favorecer la posibilidad de un tercer espacio, donde sea posible construir opciones deseables que puedan plantearse como alternativas a las que -de otra manera- la realidad seguirá imponiendo. (García Guadilla, 2003, p. 35).

La tensión entre ambos enfoques y, sobre todo, una polarización que desde la educación superior condujera a radicalizar una posición de rechazo purista, haría emerger el riesgo de ser desvirtuadas tanto en su naturaleza como en su finalidad, las distintas esferas de actividad académica en las que, según se ha indicado anteriormente, la internacionalización de la educación superior puede representar una importante opción programática y de política para el desarrollo académico e institucional.

La dimensión de internacionalización no representa únicamente un instrumento para la modernización de las estructuras de funcionamiento de la educación superior. Aporta también una heurística que permite repensar la función propiamente dicha de la educación superior, en tanto vinculada 
con la sociedad y en articulación con la búsqueda programática de nuevos horizontes y umbrales de pensamiento, de reflexión y de acción.

\section{III. Ámbitos para una política de internacionalización en la educación superior}

Cuatro ámbitos del quehacer académico en una institución de educación superior pública figuran como lugares propicios para el desarrollo de una política de internacionalización. Estos son: el intercambio académico, los planes de estudio y programas, la gestión institucional, y las redes académicas interuniversitarias e internacionales. De los cuatro, este último es uno de los ámbitos que presenta una mayor cualificación durante los últimos años, y que adquiere cada vez una relevancia mayor conforme la sociedad del conocimiento y la globalización profundizan y amplían el alcance de sus implicaciones en términos estructurales para la educación superior.

\section{III.1. Intercambio académico}

Como parte de una interacción innovadora en relación con las prácticas académicas tradicionales, la internacionalización de la educación superior introduce un dinamismo inédito en el marco de las acciones de movilidad o del intercambio académico. Los escenarios de la globalización y de la sociedad del conocimiento implican que las iniciativas y orientaciones del intercambio académico, adopten ahora otros contenidos y otras orientaciones, sustentados esta vez en la organización de las acciones a partir de la definición de una política institucional codificada en función del logro de mayores grados de integración y de integralidad programática y sistémica.

El intercambio académico, en tanto escenario que favorece la apertura de múltiples y diversos espacios para el relacionamiento académico y del proyecto de formación, adquiere también una relevancia central como dispositivo que permite engarzar con las demandas y desafíos histórico/sociales y humanísticos que en el presente la globalización y la sociedad del conocimiento involucran para la educación superior.

La complejidad de la vida social y profesional es tal que las lenguas, las formas de vida y los discursos ejercen una profunda influencia formativa. Sin embargo, en medio de toda esta diferenciación cognitiva, podemos seguir sosteniendo que, siendo como son las fuerzas sociales, se tiende a favorecer ciertas formas de pensamiento y acción, mientras que otras son menoscabadas o directamente dejadas de lado. Estas predilecciones y desestimaciones también se están abriendo camino en la educación superior. (Barnett, 2001, pp. 36/37).

Atendiendo a estas tendencias y a las nuevas configuraciones y reformulación que involucran, el intercambio académico demanda ser asumido bajo el estatuto de un dispositivo que cualifica las condiciones, contenidos, modalidades y sentidos del proyecto de formación, de conformidad con los procesos de transformación que se abren paso y perfilan desde la matriz curricular de los programas y los planes de estudios. "Es aquí donde la labor de intercambio toma una importancia vital, desde las múltiples facetas que tiene la actividad, siempre con la visión de coadyuvar al desarrollo de las propias instituciones." (Cortés, 1996, p. 25).

Además del desarrollo, ampliación y diversificación de sus contenidos tradicionales, el intercambio académico pasa igualmente a ser definido ahora en términos de una concepción axiológica inédita; está llamado a prestar contribución significativa a la promoción y enriquecimiento de una sensibilidad intercultural propositiva y aportante, como referente que da su identidad a los procesos del proyecto de formación y que, en general, interpela a los actores diversos que conforman la comunidad universitaria. 
El problema de la integración de la vida cultural se convierte en el problema de hacer posible que las personas que habitan en mundos distintos tengan un impacto genuino y recíproco unos sobre otros. Si es cierto que, en la medida en que exista una conciencia general, ésta ha de consistir en la interacción de una caótica multitud de visiones no del todo conmensurables, entonces la vitalidad de esa conciencia dependerá de la creación de las condiciones bajo las que pueda darse esa interacción. (Geertz, 1984, p. 190).

En el marco de las condiciones estructurales y de los requerimientos programáticos que los escenarios societales y culturales del presente establecen para las instituciones de educación superior, el intercambio académico está interpelado también y convocado a favorecer alternativas para que estas instituciones puedan enriquecer los procesos académicos, allende las implicaciones de iniciativas circunscritas a acciones periféricas o de carácter restringidamente particularizado. En este sentido y como parte de una apropiada política institucional de internacionalización, el intercambio académico aboga por la configuración de alternativas de formación y de desarrollo académico que, tanto para los estudiantes como para los académicos en general, promueve la apertura de vías diversas de acceso a la experiencia de la interacción proactiva y prospectiva con otras comunidades académicas, en relación con otros estilos de vida y con otras prácticas e imaginarios culturales.

\section{III.2. Planes de estudio y programas}

Con la globalización y la sociedad del conocimiento, los planes de estudio y programas se constituyen en uno de los ámbitos fundamentales donde la intervención de los actores externos presenta cada vez mayores incidencias. Este representa asimismo quizá el ámbito académico donde cada vez con mayor relevancia, se perfila como clave la internacionalización en el contexto de la educación superior pública.
Más allá de disposiciones tales como incorporar la enseñanza de un idioma en el plan de estudios, el proyecto de formación acusa ahora una tensión estructural que deriva a partir de los requerimientos de flexibilización de los perfiles profesionales y de la recurrida interlocución de competitividad que establecen como horizonte deseado los escenarios del mercado a escala nacional e internacional. Para las instituciones de educación superior, tal tensión se configura como una demanda por armonizar su proyecto de formación en procura de adecuar "los sistemas educativos a las exigencias productivas y a la competitividad global basada en la incorporación de progreso técnico". (CEPAL, 2000, p. 95). En la óptica de la CEPAL:

Para los países de la región esto implica impartir formación para un desarrollo productivo que debe tomar en consideración las destrezas propias de la sociedad de la información y el conocimiento. Este desafío está lejos de recibir una respuesta adecuada de nuestros sistemas educacionales, en los que el cambio de orientación y estilos pedagógicos todavía es incipiente. (Comisión Económica para América Latina y El Caribe, 2000, pp. 95/96).

No obstante y, a propósito, vale tomar en cuenta las especificidades que en su enfoque, É. Morin destaca de manera general como las condiciones en las que se debe sustentar ahora la identidad curricular del proyecto de formación en la educación superior:

Debemos comprender que hay condiciones bioantropológicas (las aptitudes del cerebro $\leftrightarrow$ mente humano), condiciones socioculturales (la cultura abierta que permite los diálogos e intercambios de ideas) y condiciones noológicas (las teorías abiertas) que permiten "verdaderos» interrogantes, esto es, interrogantes fundamentales sobre el mundo, el hombre y el conocimiento mismo. (Morin, 2001b, p. 31).

Estas condiciones bioantropológicas demandan ser dilucidadas desde los escenarios del plan de estudios y los programas, a través de un ejercicio programático en el que la movilidad estudiantil y docente, la participación y el encuentro en el aula con 
estudiantes extranjeros (Gacel, 2000b, p. 87), la apertura y desarrollo de la sensibilidad disciplinar a la diversidad cultural y a las distintas formas y modalidades inter, trans y multidisciplinarias del conocimiento, etc., contribuyen a forjar y enriquecer las condiciones diversas y complejas de la actividad académica institucional.

Desde una orientación como la que promueve la UNESCO: "En la vida de los seres humanos, 'ser' significa conocer y reconocer. La educación facilita dotarles del carácter humano y de los elementos necesarios para su individualidad y libertad." (Figueroa, 2003, p. 7). En el plano del proyecto de formación en educación superior, en consecuencia, podría afirmarse: "La valoración de la inteligencia desde el acto de convivir explica el desarrollo de la preocupación por educar a los jóvenes para que sean buenos ciudadanos, es decir, para que sepan participar debidamente en la vida libre de la comunidad." (Tierno, 1969, p. 45). Por supuesto, es claro que ni la "vida libre" ni la comunidad constituyen entidades fijas dotadas de especificidades unilateralmente decididas de una vez y para siempre. Antes bien, configuran en el horizonte de lo posible, la necesidad de una participación ciudadana orientada en pro de la construcción permanente y abierta tanto de la una como de la otra.

Así, el intercambio, pero fundamentalmente el intercambio inducido a partir del ethos del diálogo, ha de ser entendido como tal en razón de un proceso de participación y recíprocamente constructivo. El diálogo "supone acción en multitud de sentidos." (Tierno, 1969, p. 48). En su relación con los programas y planes de estudio se aboga ahora en la educación superior por una formación basada en el respeto a las diferencias, la reciprocidad, el sentido y conciencia de la diversidad, la solidaridad y la equidad en sus múltiples implicaciones y requerimientos.

La naturaleza genuina de una construcción académica sustentada en relaciones de diálogo se pone de manifiesto "cuando expresa el proceso de la sociedad en situación de fluidez, participación, apertura y conciencia de la realización en el devenir." (Tierno, 1969, p. 83).

"La característica principal del hombre-masa no es la brutalidad y el atraso, sino su aislamiento y su falta de relaciones sociales normales." (Arendt, 1974, p. 398). Por "relaciones sociales normales" puede entenderse en este contexto relaciones sociales propositivas, la consideración de la dimensión del sujeto a diferencia del proyecto de tecnoburocratización de los individuos, tal como señala É. Morin que es predominante en los sistemas contemporáneos de organización de la sociedad. De conocimiento común es que un individuo puede llegar a alcanzar el desarrollo de altos grados de experticia en la realización de cualquier actividad técnica o tecnológica, que no por eso queda necesariamente exonerado de ser una pieza acrítica subrogada en la maquinaria de la masificación tecnoburocratizada del trabajo. (Morin, $2001^{\text {a }}$, p. 28).

Desde esta perspectiva, incluso una formulación elaborada hace ya casi un siglo por J. Dewey adopta en la actualidad una renovada vigencia. En su preocupación por señalar lo que él considera como las deficiencias características de un proceso de formación educativa que se desplegara únicamente atendiendo a las coordenadas de lo endógeno, Dewey alertaba sobre "el peligro constante de que el material de la instrucción sistemática sea meramente el tema de estudio de las escuelas, aislado de los temas de las experiencias de la vida." (Dewey, 1997, p. 19). En su opinión, con ello no se lograría sino quedar a expensas de "perder de vista los intereses sociales permanentes". Bajo el concepto de una formación basada en el "aprender a aprender" -que en la actualidad resuena por doquier cuando se trata de la cuestión del aprendizaje-, Dewey añadía además en su momento lo siguiente:

Una sociedad que es móvil, que está llena de canales para la distribución de un cambio que ocurra en 
cualquier parte tiene que procurar que sus miembros sean educados para la iniciativa y la adaptabilidad personales. De otro modo éstos se verán abrumados por los cambios a que estarán sometidos y cuya significación o conexión no percibirán. El resultado será una confusión, en la que unos pocos se apropiarán de los resultados de las actividades ciegas y dirigidas externamente de los demás. (Dewey, 1997, p. 82).

En lo referente a los programas y planes de estudio, vale destacar la existencia de una tensión que se configura entre las "demandas de la sociedad" y lo que la educación superior define como los elementos sustantivos que deben formar parte de su proyecto educativo. Esta tensión puede ser recuperada de manera significativa y proactiva; ella "exige de la teoría pedagógica una concepción más clara del sentido de lo "social", como una función y prueba de la educación, de la que se ha alcanzado hasta ahora." (Dewey, 1997, p. 90). En un nivel estructural y de nuevo en la perspectiva de É. Morin:

Existe un problema capital, (...) la necesidad de promover un conocimiento capaz de abordar los problemas globales y fundamentales para inscribir allí los conocimientos parciales y locales. // La supremacía de un conocimiento fragmentado, según la disciplina, impide a menudo operar el vínculo entre las partes y las totalidades y debe dar paso a un modo de conocimiento capaz de aprehender los objetos en sus contextos, sus complejidades, sus conjuntos. (...) Es necesario enseñar los métodos que permiten aprehender las relaciones mutuas y las influencias recíprocas entre las partes y el todo en un mundo complejo. (Morin, 2001b, p. 14).

L. Garay, a propósito de esta tensión, señala que en las instituciones educativas "el conflicto constituye un trasfondo permanente; fuente de dificultades y sufrimiento, pero también del que nacen el cuestionamiento, la movilización de lo instituido por lo instituyente, el descubrimiento de nuevas formas y de nuevas matrices de sentido." (Garay, 1996, p. 140).

A efecto de las transformaciones en los planes de estudio y del proyecto de formación en la educación superior, en consecuencia, esta labor no se puede desvincular del campo institucional entendido en sentido amplio. (Garay, 1996, p. 136). Asimismo, los intereses y demandas que plantean en su articulación compleja los diferentes sectores de la sociedad, abarcan otros elementos de mayor amplitud que los que establecen las demandas de coyuntura del sector productivo y del mercado laboral. Demandas estas que, por lo general, se establecen a partir de "los sectores más dinámicos" de la economía, los vinculados al mercado internacional y a la globalización y cuyas pautas de orientación están dadas por el principio de la competitividad transnacionalizada. Estas condiciones acarrean para la educación superior desafíos estructurales; las obligan a configurar nuevas referencialidades y a establecer nuevos principios de relacionamiento interinstitucional. En los umbrales de estos nuevos espacios y nuevas articulaciones, los planes y programas plantean requerimientos de atención urgente e insoslayable.

\section{III.3. Gestión institucional}

Un tercer ámbito naturalmente implicado en la perspectiva de una política de internacionalización de la educación superior es el de la gestión institucional. Este ámbito se encuentra estrechamente articulado con los dos anteriores y, por definición, les sirve de soporte.

El espacio de la gestión remite a las disposiciones por generar una mayor articulación programática y estratégica con y entre los diferentes otros componentes que constituyen el entramado del espacio institucional: intercambio académico, desarrollo internacionalizado de los planes de estudio y los programas, redes académicas (con mucho énfasis en investigación y en posgrado, pero también en el propio intercambio académico), la misma formación del personal administrativo.

A propósito de la gestión, el Banco Interamericano de Desarrollo (BID), en su documento La educación superior en América Latina y el Caribe, señala que, en 
su gran mayoría, las instituciones de educación superior de América Latina presentan deficiencias en materia de definición y gestión de políticas para un adecuado desarrollo en diferentes ámbitos, especialmente en los de la docencia y la investigación. (Castro y Levy, 1997, p. 10).

Correlativamente, como insuficiencia programática y de la política académica, las acciones para el fortalecimiento de los perfiles docentes y de la formación en investigación de los académicos presentan igualmente una orientación de gestión que es omisa en aprovechar las diversas potencialidades que el intercambio académico puede favorecer y llegar efectivamente a generar.

Más allá de la referencialidad epistemológica que pueda asumir y de la teleología que de sustento a tal enfoque, no solo en el caso del BID sino también en el de otros organismos multilaterales, el tema de la gestión en la educación superior no deja de conducir en la actualidad a la identificación de una problemática diversa. En su conjunto, las políticas y cursos de acción que organizan la gestión en función de los derroteros del desarrollo académico e institucional, encuentran en las situaciones estructurales predominantes, escenarios y situaciones desafiantes y de una gran complejidad.

Los diversos flujos que vemos (de objetos, personas, imágenes y discursos) no son coetáneos, convergentes, isomórficos o espacialmente congruentes. Mantienen relaciones de disyunción. Esto quiere decir que las vías o vectores seguidos por estos diversos fenómenos tienen diferentes velocidades, diferentes ejes, diferentes puntos de origen y fin y diferentes relaciones con las estructuras institucionales en diferentes naciones, regiones o sociedades. Por otro lado, estas disyunciones en sí mismas precipitan diversos tipos de problemas y fricciones en diferentes situaciones locales." (Appadurai, 1999, p. 4).

R. Barnett encuentra que, tal como sucede en el campo y dominios del conocimiento, así también en la gestión institucional actual de la educación superior se configura y tiende a prevalecer una impronta de comportamientos y de prácticas rígidas y unidimensionales. (Barnett, 2001, pp. 32/33). Estas tienen por característica en su incidencia sobre la cultura organizacional, un endurecimiento o cristalización de las prácticas organizacionales en la forma de "habitus" (Bourdieu, 2000, pp. 23/40), o de lugares comunes idiosincrásicos. Como resultado de ello, prevalece la puesta en suspenso y limitada la posibilidad de incorporar modalidades de mayor interacción, flexibilidad y dinamismo.

De este modo, en consecuencia, los enunciados emblemáticos que identifican a las instituciones de educación superior, por ejemplo, la condición de la educación superior como bien público, tienden a ser reducidos a una simple consigna y, como tal, a perder su potencialidad y su fuerza heurística como resultado de la incidencia de lo que puede ser definido como las inercias constitutivas de la rigidez burocrática y tecnocrática. Como si, ante la necesidad de romper con lo que, en su perspectiva, E. Remedi denomina como la "hipertrofia de la memoria institucional", las instituciones de educación superior también requirieran, en la dimensión de sus actividades de gestión, recurrir al uso de dispositivos panópticos (Foucault, 1999, p. 228) para controlar los cambios y, de este modo, asegurar su "estabilidad" y ponerse a salvo de las dinámicas y cursos de acción que implican los procesos de cambio.

La educación superior institucionalmente involucra una perspectiva de valores, con mucha frecuencia un compromiso con el desarrollo democrático de la sociedad, así como una sensibilidad y una proyección de humanidad que le son características.

La educación superior constituye una institución única en la sociedad moderna. Convocada por la sociedad para cumplir con una variedad de funciones, finalmente llega a ser más que la suma de las partes. Cada uno de sus aspectos está teñido por los otros. Es posible que el ensamble entre las partes sea endeble (...); sin embargo, las partes funcionales están ensambladas y existe una interacción dinámica entre ellas. (Barnett, 2001, p. 50). 
De cara a los imaginarios y dispositivos que hacen prevalecer las condiciones de "hipertrofia de la memoria institucional", corresponde a la gestión académica e institucional proveer las condiciones y mecanismos para recuperar y enriquecer el dinamismo de las interacciones y generar la cultura y los ambientes necesarios para un óptimo aprovechamiento de las potencialidades y alternativas de fortalecimiento del proyecto académico institucional. Tal como lo señala L. Garay, en la actualidad, la educación superior requiere impulsar procesos que posibiliten "el advenimiento de imaginarios instituyentes como fuente de nuevas simbolizaciones y nuevos sentidos." (Garay, 1996, pp. 155/156).

Lo mismo que para los programas y los planes de estudio, la gestión institucional, orientada a la adopción de una política de internacionalización precisa y apropiada, aporta la posibilidad de abrir un espacio en el que la institución puede desarrollar acciones de muy diversa naturaleza, articuladas en función de dar apoyo y un sentido apropiado a las dinámicas de la transformación y del cambio, en síntesis, de la renovación académica e institucional.

En el marco de la gestión, cabe precisar, finalmente, que la política institucional de internacionalización aporta la oportunidad de volver a tejer las relaciones dinámicas y preactivas entre los diferentes actores (administrativos, estudiantiles, docentes, etc.) en el interior de la institución; también, contribuye a repensar y reconfigurar los mecanismos, sentidos y objetivos de la gestión, en su perspectiva de acción interinstitucional y en interacción con instituciones de otros países y regiones del mundo.

\section{III.4. Redes académicas}

Un cuarto y último aspecto que interesa tocar en la presente oportunidad y que, en su lugar, adquiere también cada vez mayor relevancia en los escenarios de la educación superior, es el de la articulación y establecimiento de redes académicas internacionales. (PNUD, 2001, pp. 4/5).

El aspecto esencial está constituido por una aceleración sin precedentes del ritmo de creación, acumulación y sin duda también de depreciación del conocimiento. (...). Un nuevo tipo de institución participa fundamentalmente en este fenómeno. Se trata de las comunidades de conocimientos: redes de individuos cuyo objetivo fundamental es la producción y la circulación de saberes nuevos y que ponen en relación a personas que pertenecen a entidades diferentes 0 incluso rivales. (David y Foray, 2002, p. 7).

Las redes académicas no representan un modelo nuevo en el marco de las políticas y los cursos de acción de la educación superior. No obstante, según señala $\mathrm{H}$. Ayarza, "nunca antes fueron tan numerosas como en la actualidad, ni tuvieron la importancia que tienen hoy en día en la perspectiva de la cooperación universitaria internacional." (Ayarza, 1997, p. 79).

Esta modalidad de interacción académica adquiere un lugar destacado, sobre todo a partir de los procesos de integración regional y macrorregional originados durante los últimos años, y que, en la perspectiva de la internacionalización, procuran constituirse en una de las respuestas privilegiadas que las instituciones de educación superior dan a las tendencias de la globalización y de la sociedad del conocimiento. La importancia de las redes académicas la pone de relieve, por ejemplo, el ya anteriormente mencionado Programa Alfa de la Unión Europea. Pero existen también otras diversas experiencias. Así, el Programa de Administración Institucional de la Educación Superior (IMHE), de la Organización para la Cooperación y el Desarrollo Económicos (OCDE), tiene su origen específicamente sobre la base del principio según el cual, "la internacionalización y su contribución a la educación superior están adquiriendo más reconocimiento en todo el mundo, tanto en los países en vías de desarrollo como en los desarrollados." (Knight y de Witt, 2001, p. 56).

Las iniciativas para la constitución de redes en relación con la internacionalización de la educación superior también 
están presentes en el marco del Tratado de Libre Comercio de América del Norte. En este caso, registra como tal la iniciativa de la Fuerza Trilateral de Discusión en Educación Superior, entre cuyas disposiciones figura la de crear la red NADERN con la finalidad de prestar atención al tema de la internacionalización en los ámbitos de la investigación y de la educación a distancia, como parte de las acciones que involucran una mayor interacción entre los sistemas de educación superior de Canadá, Estados Unidos y México. (Didou, 2000b, pp. 77/86).

Igualmente, por su parte, en Centroamérica, a partir de 1995, en el escenario de integración regional del que da cuenta la educación superior pública en el contexto del PIRESC II del CSUCA, se cuenta con la constitución de los sistemas universitarios regionales que, operando bajo el concepto de redes académicas centroamericanas, han permitido llevar adelante un intenso proceso de trabajo interinstitucional asociativo y de colaboración recíproca y solidaria entre las instituciones de educación superior pública de estos países. Como resultado de esta experiencia, cabe destacar, por ejemplo, el establecimiento del Sistema Centroamericano de Evaluación y Acreditación de la Educación Superior (SICEVAES), el Sistema de Carreras y Posgrados Regionales Centroamericanos (SICAR), el Consejo Regional de Vida Estudiantil (CONREVE), así como experiencias de intercambio académico extrarregionales, tales como la que configura el Programa Mesoamericano de Intercambio Académico ANUIES/CSUCA.

La constitución de redes académicas representa para la educación superior igualmente un importante y necesario escenario para el fortalecimiento de la investigación y del posgrado. Las redes académicas en investigación y posgrado adquieren durante los últimos años una importancia creciente, cuanto más que contribuyen a que las instituciones de educación superior impulsen acciones en el sentido de desarrollar políticas y procesos apropiados que les permitan mejorar, fortalecer y actualizar el perfil académico de su planta docente, tanto como ofrecer a la sociedad una más consistente oferta de servicios educativos en las distintas dimensiones y niveles del proceso de formación.

A propósito de la importancia que adquiere la formación de posgrado, R. Lucio señala que este representa "el área de intersección entre dos sistemas claves (...): el sistema de educación superior, por un lado, y el sistema de producción científica y tecnológica, por el otro."1 En el primer sentido, la investigación y el posgrado adquieren una importancia insoslayable en la medida que las universidades están urgidas de implantar mecanismos propios que les permitan mejorar, fortalecer y actualizar el perfil de conocimientos de su planta docente, tanto como ofrecer a la sociedad la más consistente oferta de servicios educativos en este nivel de formación. En el segundo sentido, teniendo en cuenta que una de las dimensiones naturales del posgrado está constituida por la investigación, se vuelve asimismo un mecanismo que permite abrir espacios para el fortalecimiento, diversificación y ampliación de las capacidades para la producción de conocimientos científicos y tecnológicos.

En su caso, la articulación de la investigación con el posgrado favorece el fortalecimiento académico institucional, en la medida que los propios estudiantes constituyen de por sí un factor fundamental de desarrollo. A juicio de A. Appadurai, "la investigación es prácticamente sinónimo de nuestro sentido de lo que significa ser académicos y miembros de la universidad y, por lo tanto, tiene la invisibilidad de lo obvio." (Appadurai, 1999, p. 10).

En otra de sus dimensiones sustantivas, las redes académicas constituyen un ámbito de mayor sensibilidad en el cual se pueden desarrollar acciones de colaboración con el sector productivo, el sector estatal y con diversas asociaciones civiles interesadas en el desarrollo del conocimiento y la 
tecnología. Una política atenta de creación de redes académicas en este aspecto representa una importante opción a asumir y desarrollar.

En el espacio académico de redes internacionales e interinstitucionales en investigación y posgrado, la política, estrategias y mecanismos de internacionalización de la educación superior, favorece anticiparse y prevenir que el desarrollo de los esfuerzos no derive en una labor excesivamente concentrada en atender las problemáticas que plantean las necesidades locales y que, por lo tanto, se pierda de vista el horizonte más estructural, internacional y de largo alcance estratégico en las que se inscriben los desarrollos, no sólo de la ciencia y la tecnología, sino también de los programas y los planes de estudios; en general, del proyecto de desarrollo académico institucional.

\section{Conclusiones}

En su acepción académica más precisa, la internacionalización de la educación superior supone un conjunto de disposiciones de política y programáticas orientadas a contribuir con el desarrollo, la renovación y el fortalecimiento del proyecto académico institucional.

La globalización, conjuntamente con la sociedad del conocimiento, involucran para la educación superior codificaciones de gestión y académicas inéditas, frente a las cuales estas instituciones, o bien pueden quedar subrogadas o, por el contrario, hacer acopio de sus condiciones sistémicas y de identidad específicas, en procura de fortalecer sus capacidades de respuesta y de acción proactiva frente a las demandas que tales tendencias, procesos y escenarios les plantean.

La internacionalización, en su configuración académica, en consecuencia, favorece espacios y disposiciones de política y programáticas que aportan a la identificación y construcción de nuevos escenarios de relacionamiento interinstitucional, donde las instituciones participantes puedan obtener beneficios académicos e institucionales para su fortalecimiento y mejoramiento, mediante una puesta en común de los avances logrados en cada una de ellas. "Por ejemplo, la participación en programas de desarrollo internacional o asistencia técnica puede generar beneficios mutuos para todos los socios y cambios importantes en la investigación, el desarrollo curricular y la enseñanza." (Knight, 2003, p. 60).

De este modo, la política y programas de internacionalización, a través de su articulación con los componentes sustantivos que definen las preocupaciones y los intereses institucionales, (el intercambio académico, la reforma de los programas y planes de estudio, la gestión institucional, el desarrollo y fortalecimiento de la investigación y del posgrado, las redes académicas), ofrece condiciones apropiadas y representa una alternativa que práctica y potencialmente levanta perspectivas de contribución significativa en el marco de los procesos de transformación que hoy son requeridos en la educación superior.

Una universidad de excelencia que quiera mantener presencia y liderazgo en el campo de la educación superior y el desarrollo científico y tecnológico tiene como imperativo, hoy en día, incrementar sus relaciones interinstitucionales internacionales, perfilar su propio aporte al desarrollo de la comunidad internacional y aprovechar plenamente las posibilidades, tanto de perfeccionamiento, becas, como recursos que se generan en este ámbito. (Neumann, 2000, p. 13).

No obstante, la alternativa de la internacionalización tampoco puede ser ni valorada ni asumida como si se tratase de una panacea. La reconfiguración de las condiciones, propósitos, políticas y programas académicos, en síntesis, la reorganización de contenido y de desempeño del proyecto institucional de desarrollo, es de una gran complejidad y responde a múltiples y muy diversos factores. Es obvio que no existe forma de abordar estos procesos de manera apropiada, estratégica y prospectiva, a partir de una idea simplista de 
la naturaleza y características que definen a estas instituciones.

La internacionalización, lo mismo que constituye una opción de oportunidad también representa un desafío. Justamente el desafío por desarrollar la capacidad de interactuar con otras instituciones en diversas escalas -nacional, regional e internacional-, teniendo al mismo tiempo como propósito que el relacionamiento interinstitucional establecido contribuya a fortalecer y a enriquecer los propios procesos académicos y de gestión que dan cuenta del proyecto institucional de desarrollo.

\section{Nota}

1. Lucio, Ricardo, Op cit, p. 326.

\section{Referencias bibliográficas}

Appadurai, Arjun. "La globalización y la imaginación en la investigación", Revista Internacional de Ciencias Sociales, No 160, París: http:// www.unesco.org/issj/rics160/ appaduraispa.html\#aa 1999.

Arendt, Hannah. Los orígenes del totalitarismo, Madrid: Taurus, 1974.

Ayarza Elorza, Hernán. "La internacionalización de la universidad latinoamericana: algunos factores incidentes", Educación Global, $N^{\circ} 1$, Guadalajara: Asociación Mexicana para la Educación Internacional, 1997.

Barnett, Ronald. Educación superior: historia y politica. Estudios comparativos sobre la universidad contemporánea, Barcelona: Gedisa, 2001.

Bauman, Zygmunt. $2^{\text {a }}$ ed., La globalización. Consecuencias humanas, Ciudad de México: Fondo de Cultura Económica, 2001.
Bourdieu, Pierre. $3^{\text {a }}$ ed., Capital cultural, escuela y espacio social, Ciudad de México: siglo XII, 2000.

Boyle, David. "La implementación administrativa y académica de un programa de intercambio sustentable. Universidad Veracruzana-Universidad de Georgia”, Educación Global, $\mathrm{N}^{\mathrm{o}} 4$, Guadalajara: Asociación Mexicana para la Educación Internacional, 2000.

Castro, Claudio de Moura y Daniel C. Levy. La educación superior en América Latina y el Caribe. Documento de estrategia, Washington D.C.: Banco Interamericano de Desarrollo, http:// www.iadb.org/sds/doc/edu\%2D101s. pdf 1997.

Comisión Económica para América Latina y El Caribe. Equidad, desarrollo y ciudadanía. Versión definitiva, Santiago de Chile: CEPAL/Naciones Unidas, http:

//www.cepal.cl/publicaciones/ SecretariaEjecutiva/1/lcg2071/ lcg2071.pdf 2000.

Conferencia Mundial sobre la Educación Superior. Declaración mundial sobre la educación superior en el siglo XXI: visión y acción, París: Ediciones UNESCO, http:

//www.unesco.org/education/ educprog/wche/declaration_spa. htm\#declaracion 1998a.

Conferencia Mundial sobre la Educación Superior. La educación superior en el siglo XXI. Visión y acción, París: Ediciones UNESCO, 1998b.

Consejo Superior Universitario Centroamericano. "Fines de la Confederación Universitaria Centroamericana", Nueva Guatemala de La Asunción: Secretaría Permanente del CSUCA, 
http://csuca.edu.gt/CUC/finescuc. htm 2003.

Consejo Universitario. Estatuto Orgánico de la Universidad de Costa Rica, San Pedro de Montes de Oca, Costa Rica: Centro de Información y Servicios Técnicos/Consejo Universitario, Universidad de Costa Rica, 2004.

Cortés Bastida, Gilberto. "La importancia del intercambio académico nacional e internacional en el desarrollo de las instituciones de educación superior", en Ambriz Chávez, Rebeca; María Luisa González González y María Dolores Sánchez Soler (Coords.), Problemas y perspectivas del intercambio académico, Ciudad de México: ANUIES, 1996.

Chan Sánchez, Juan. "El Acuerdo General sobre el Comercio de Servicios (AGCS) y la educación superior"; en: García Guadilla, Carmen (ed.), $E l$ dificil equilibrio: la educación superior como bien público y comercio de servicios. Implicaciones del AGCS (GATS), Lima: Universidad de Lima/ Columbus, 2003.

David, Paul A. y Dominique Foray. "Una introducción a la economía y a la sociedad del saber", Revista Internacional de Ciencias Sociales, $\mathrm{N}^{\mathrm{o}}$ 171, París: Ediciones UNESCO, http://www.unesco.org/issj/rics171/ fulltext171spa.pdf 2002 .

Delors, Jacques. La educación encierra un tesoro, París: Ediciones UNESCO, http://www.unesco.org/delors/delors s.pdf 1996.

Dewey, John. Democracia y educación. Una introducción a la filosofía de la educación, Madrid: Ediciones Morata, 1997.
Didou Aupetit, Sylvie (coord.). Integración económica y políticas de educación superior: Europa, Asia Pacífico, América del Norte y Mercosur, Ciudad de México: ANUIES, 1998.

Didou Aupetit, Sylvie. "Macrorregionalización y políticas de educación superior: México ante el TLCAN", Revista de la Educación Superior, Vol. XXIX, N ${ }^{\circ}$ 3 (115), julio/septiembre, Ciudad de México, ANUIES, 2000a.

Didou Aupetit, Sylvie. Sociedad del conocimiento e internacionalización de la educación superior en México, Ciudad de México: ANUIES, 2000b.

Feldman, Maryann P. "La revolución de internet y la geografía de la innovación", Revista Internacional de Ciencias Sociales, $\mathrm{N}^{\circ}$ 171, París: Ediciones UNESCO http: //www.unesco.org/issj/rics171/ fulltext171spa.pdf 2002 .

Figueroa R., Federico. "Encuentro Regional sobre Educación Superior de Pueblos Indígenas de América Latina"; en: Instituto Internacional de la UNESCO para la Educación Superior en América Latina y el Caribe, La educación superior indígena en América Latina, Caracas: IESALC-UNESCO, 2003.

Foucault, Michel. Estrategias de poder, Barcelona: Paidós Ibérica, 1999.

Fransson, Lars. "Developing a policy program for internationalization: the case of Uppsala University", Educación Global, $\mathrm{N}^{\mathrm{o}}$ 4, abril, Guadalajara: Asociación Mexicana para la Educación Internacional, 2000.

Gacel Ávila, Jocelyne. La internacionalización de las universidades mexicanas. 
Políticas y estrategias institucionales, Ciudad de México: Dirección de Servicios Editoriales de la ANUIES, 2000a.

Gacel Ávila, Jocelyne. "Internacionalización de la educación superior: políticas y estrategias institucionales", Educación Global, N 4, abril de 2000, Guadalajara: Asociación Mexicana para la Educación Internacional/Editorial Gráfica Nueva, 2000b.

Garay, Lucía. "La cuestión institucional de la educación y las escuelas. Conceptos y reflexiones"; en: Butelman, Ida, Pensando las instituciones. Sobre teorías y prácticas en educación, Buenos Aires: Paidós, 1996.

García Guadilla, Carmen. "Acuerdo general de comercialización de servicios educativos (GATS) y educación superior en América Latina. Algunas ideas para contribuir a la discusión”, Lima: Convención de Universidades miembros de Columbus, http://www. columbus-web.com/es/partej/gatsyal. doc 2002 .

García Guadilla, Carmen. "Balance de la década de los '90 y reflexiones sobre las nuevas fuerzas de cambio en la educación superior"; en: Mollis, Marcela (comp.), Las universidades en América Latina: ¿̇reformadas o alteradas?, Buenos Aires: CLACSO, 2003.

Geertz, Clifford. Conocimiento local. Ensayos sobre la interpretación de las culturas, Barcelona: Paidós, 1984.

Hernández Guach, Fernando. "El interés por la educación y la formación en los tratados de la UE", Revista Sócrates, $\mathrm{N}^{\mathrm{o}} 3$, junio de 2002, [Recuperado el 5 de agosto de 2005], Madrid, http:// wwwn.mec.es/programas-europeos/ docs/revista_3.pdf 2002.
Horkheimer, Max y Theodor W. Adorno. $4^{\mathrm{a}}$ ed., Dialéctica de la ilustración. Fragmentos filosóficos, Madrid: Trotta, 2001.

Info Point Europa. "Programa SOCRATES", [Recuperado el 5 de agosto de 2005], http://www.dicoruna.es/ipe/ayudas/ JuventudEduFormacion/educacion/ socrates/programa... La Coruña. 2005 .

Internacional de la Educación. La OMC y la Ronda del Milenio: los retos de la educación pública. Cuestiones de interés común para educadores y trabajadores del sector público, Internacional de la Educación, http: //www.ei-ie.org/pub/spanish/ spbei-psi wto.html 2000.

Knight, Jane. "La internacionalización de la educación superior", en Organización para la Cooperación y el Desarrollo Económicos (2001), Calidad e internacionalización de la educación superior, ANUIES, Ciudad de México. 2001.

Knight, Jane. "Comercialización de servicios de educación superior: implicaciones del GATS", The Observatory on Borderless Higher Education, http://www.columbus-web.com/es/ partej/reportgats.doc 2002.

Knight, Jane. "Comercialización de servicios de educación superior: implicaciones del AGCS"; en: García Guadilla, Carmen (ed.), El difícil equilibrio: la educación superior como bien público y comercio de servicios. Implicaciones del AGCS (GATS), Lima: Universidad de Lima/ Columbus, 2003.

Knight, Jane y Hans de Wit. "Introducción al proyecto y proceso de IQRP", en Organización para la Cooperación y 
el Desarrollo Económicos, Calidad $e$ internacionalización de la educación superior, Ciudad de México: ANUIES, 2001.

Lucio, Ricardo. "Políticas de posgrado en América Latina", en: Kent, Rollin (comp.), $2^{\mathrm{a}}$ ed., Los temas críticos de la educación superior en América Latina en los años noventa. Estudios comparativos, Ciudad de México: Facultad Latinoamericana de Ciencias Sociales/Universidad Autónoma de Aguascalientes/Fondo de Cultura Económica, 2002.

Mollis, Marcela (comp.). Las universidades en América Latina: ¿reformadas o alteradas? La cosmética del poder financiero, Buenos Aires: Consejo Latinoamericano de Ciencias Sociales, 2003.

Morin, Édgar. $6^{\text {a }}$ ed., El paradigma perdido. Ensayo de bioantropología, Barcelona: Kairós, 2000.

Morin, Édgar. (3 ${ }^{\mathrm{a}}$ ed.), El Método IV. Las ideas: su hábitat, su vida, sus costumbres, su organización, Madrid: Cátedra, 2001a.

Morin, Edgar. Los siete saberes necesarios para la educación del futuro, Ciudad de México: Librería El Correo de la UNESCO, 2001b.

Morin, Edgar. El método V. La humanidad de la humanidad. La identidad humana, Madrid: Ediciones Cátedra, 2003.

Mungaray, Alejandro y Juan Manuel Ocegueda. "La educación superior en la integración económica de América del Norte, en Didou Aupetit, Sylvie (coord.), Integración económica y políticas de educación superior: Europa, Asia Pacífico, América del
Norte y Mercosur, Ciudad de México: ANUIES, 1998.

Neave, Guy. Educación superior: historia y política. Estudios comparativos sobre la universidad contemporánea, Barcelona: Gedisa, 2001.

Neumann, Raúl A. “Tendencias y políticas en el proceso de internacionalización de las universidades chilenas"; citado en: Gacel-Ávila, Jocelyne: La internacionalización de las universidades mexicanas: políticas y estrategias institucionales, Ciudad de México: ANUIES, 2000.

OECD. Repaso a la enseñanza: Indicadores de la OECD - Edición 2004, París: Dirección de Relaciones Públicas y Comunicaciones de la OECD, http://www.oecd.org/ dataoecd/33/24/33713498.pdf 2004.

Oficina de Relaciones Internacionales y Cooperación Externa. "Unión Europea: Programa América Latina Formación Académica”, San Pedro de Montes de Oca: Universidad de Costa Rica, http://www.oaice.ucr.ac.cr/ programa_alfa.htm 2004.

Ottone, Ernesto. "La apuesta educativa en América Latina", Revista de la CEPAL, Número Extraordinario, Santiago de Chile:http://www.cepal.cl/ publicaciones/SecretariaEjecutiva/7/ lcg2037/ottone.htm 1998.

Panizzi, Wrana María. "La educación superior como "servicio comercial": ¿Desafío o amenaza?”; en: García Guadilla, Carmen (ed.), El difícil equilibrio: la educación superior como bien público y comercio de servicios. Implicaciones del AGCS (GATS), Lima: Programa Columbus, 2003.

Programa de las Naciones Unidas para el Desarrollo. Informe sobre desarrollo 
humano 2001. Poner el adelanto tecnológico al servicio del desarrollo humano, Ciudad de México: Ediciones Mundi-Prensa, http://www.undp.org/ hdr2001/spanish/spidhtod.pdf 2001.

Remedi Allione, Eduardo. "La institución: un entrecruzamiento de textos", Ciudad de México: DIE/CINVESTAV, 2003.

Ribeiro Durham, Eunice. "Introducción: los estudios comparativos de la educación superior en América Latina", en: Kent, Rollin (comp.), $2^{\mathrm{a}}$ ed., Los temas críticos de la educación superior en América Latina en los años noventa. Estudios comparativos, Ciudad de México: Facultad Latinoamericana de Ciencias Sociales/Universidad Autónoma de Aguascalientes/Fondo de Cultura Económica, 2002.

Robinet, André. Mitología, filosofía y cibernética, Madrid: Tecnos, 1982.

Tierno Galván, Enrique. Razón mecánica y razón dialéctica, Madrid: Taurus, 1969.

Torres, Jurjo. $4^{\mathrm{a}}$ ed., Globalización e interdisciplinariedad: el currículum inte- grado, Madrid: Ediciones Morata, 2000.

Universidad de Lima. "El difícil equilibrio: la educación superior como bien público y comercio de servicios. Orientación para una indagación sobre el tema", http://www.columbus-web.com/es/ partej/gatslima.html 2002.

Valaskakis, Kimon. "La globalización como teatro: nuevo escenario, nuevos actores, nuevo guión”, Revista Internacional de Ciencias Sociales, $\mathrm{N}^{\circ}$ 160, París: http://www.unesco.org/issj/ rics160/valaskakisspa.html\#vv 1999.

Van Der Donckt, Pierre. "El Colegio de las Américas", Educación Global, $\mathrm{N}^{\circ} 1$, Guadalajara: Asociación Mexicana para la Educación Internacional, 1997.

Vigil Ávalos, Carlos. "La universidad latinoamericana ante los nuevos escenarios de la región”; en: Seminario Internacional: La universidad latinoamericana ante los nuevos escenarios de la región (1995), La universidad latinoamericana ante los nuevos escenarios de la región, UDUAL/Universidad Iberoamericana, Ciudad de México. 1995. 
